\title{
Le monde du « si » : idéologies incertaines dans Comme il vous plaira
}

\section{Marie-Thérèse Jones-Davies}

Marie-Thérèse Jones-Davies (éd.)

\section{(2) OpenEdition Journals}

\section{Édition électronique}

URL : http://journals.openedition.org/shakespeare/432

DOI : $10.4000 /$ shakespeare.432

ISSN : 2271-6424

\section{Éditeur}

Société Française Shakespeare

\section{Édition imprimée}

Date de publication : 1 novembre 1981

Pagination : 51-68

ISBN : 2-86433-016-4

\section{Référence électronique}

Marie-Thérèse Jones-Davies, « Le monde du « si » : idéologies incertaines dans Comme il vous plaira », Actes des congrès de la Société française Shakespeare [En ligne], 3 | 1981, mis en ligne le 01 novembre 2007, consulté le 20 janvier 2020. URL : http://journals.openedition.org/shakespeare/432 ; DOI : $10.4000 /$ shakespeare.432 


\title{
THEATRE ET IDEOLOGIES :
} Marlowe, Shakespeare

\author{
DIRECTEUR DE LA PUBLICATION \\ M.T. Jones - Davies
}

JEAN TOUZOT Libraire - Editeur

38 , rue Saint-Sulpice 75278 PARIS CEDEX 061982 
1982 Jean Touzot Libiaire-Editeur, Paris.

Reproduction et traduction mzme partielle interdite.

Tous droits réservts potir lous pays

y compris i'U,R.S.S. et les pays scandinaves

ISBN 2-86433-016-4 


\title{
AVANT-PROPOS
}

L'opposition soulignée par Horace entre éthique et esthétique Aut prodesse volunt aut delectare poetae - que les Elisabéthains aiment à évoquer, pourrait être le lien qui rattache les unes aux autres les communications, rencontres et table ronde de notre troisième congrès (1981) sur le thème : "Théátre et Idéologies: Marlowe, Shakespeare.» Toutefois, le résultat de nos échanges montre un dépassement possible de l'alternative éthique / esthétique, puisqu'il met en valeur l'insertion d'idéologies susceptibles de transformer la fonction même de la littérature.

Les pièces analysées dans le contexte de leur temps révèlent de multiples aspects de la vision du monde et de la place que l'homme $y$ occupe, tels que les projette le théâtre anglais au tournant du XVIe et au début du XVIIe siècle.

Une exploration du langage et de la pensée des deux grands dramaturges aboutit à un commentaire sur les courants philosophiques ou religieux, sur les idées parfois contradictoires et sur la curiosité intellectuelle jamais assouvie qui se font jour à travers la rhétorique de la scène et le jeu dramatique, du Faust de Marlowe à La Tempéte de Shakespeare.

\author{
M.T. jones-Davies
}

Présidente de la Société Française Shakespeare 
SOCIETE FRANCAISE SHAKESPEARE Actes de Congrès.1981

\section{THEATRE ET IDEOLOGIES : Marlowe, Shakespeare}

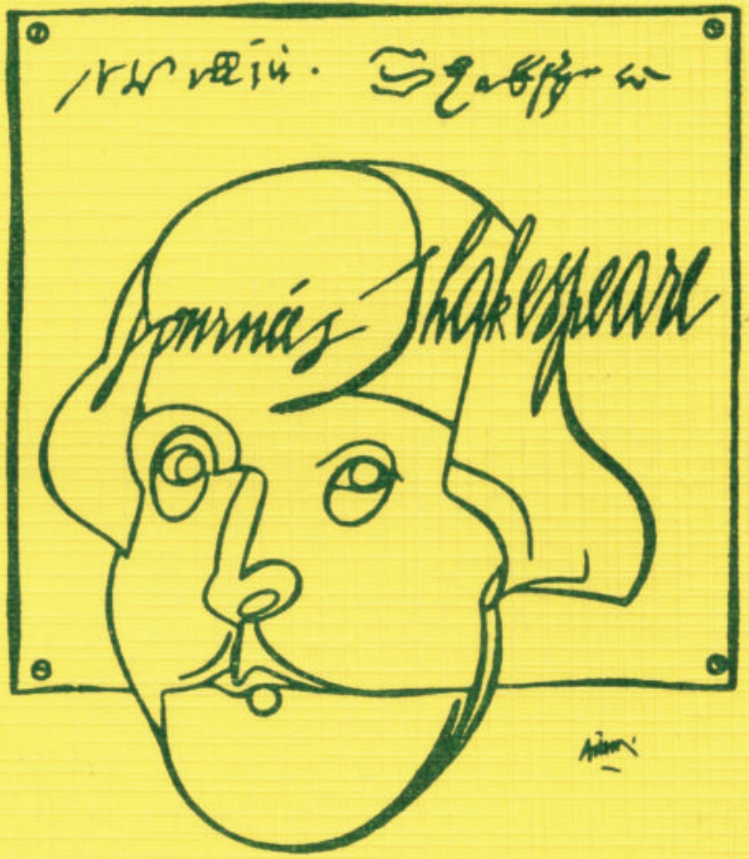

DIRECTEUR DE LA PUBLICATION M.T. Jones - Davies

JEAN TOUZOT Libraire - Editeur 38 , rue Saint-Sulpice 75278 PARIS CEDEX 061982 
Illustration de la couverture : affiche de Valerio Adami, spécialement conçue pour les Journées Shakespeare 1979 au Centre Georges Pompidou 


\section{TABLE DES MATIERES}

M.T. JONES-DAVIES Avant-propos

Théâtre et Idéologies: Marlowe, Shakespeare

S. TRUCHET

The Alchemy of Beauty: aesthetic principles in I Tamburlaine and their relationship to the hermetic ideology.

D. PRUDHOMME

L'idéologie du Juif de Malte et du Marchand de Venise.

D. GOY-BLANQUET

De Hall à Shakespeare : quelques glissements idéologiques opérés par la dramatisation dans Henry $I V$.

M.T. JONES-DAVIES Le Monıde du «si»: Idéologies Incertaines dans Comme il vous Plaira.

G. VENET

Temps et Idéologie : Marlowe, Shakespeare.

J. RICHER

Les Sept Caractères et les Quatre Tempéraments dans Jules César de Shakespeare.

F. LAROQUE

En marge de l'Idéologie : Antimasque et Grotesque dans le Dr Faustus et La Tempête.

A. LECERCLE-SWEET Conscience et Méconnaissance dans Edward The Second de Marlowe.

M.A. CONEJERO Shakespeare's Aesthe tic Scheme. (Extrait).

P.STEWART

Shylock, Shakespeare's Alien.

(Royal Shakespeare Company)

J.RUSSELL BROWN The Romantics' Shakespe are.

R. GILL

The Christian Ideology of Dr Faustus.

J. JACQUOT

Marlowe : de quelques problèmes d'interprétation. 
Rencontres entre Universitaires et Gens de Théâtre

Vendredi 4 décembre 1981
- Stuart Seide :Présentation du

Songe d'une nuit d'Eté. Mise en scène pour le Théâtre de Chaillot janvier 1982

- A propos d'Edouard (II) de

Bernard Turle

Bernard Turle : Métamorphoses de l'auteur et de l'écriture dramatiques. Intervention de Colin Harris (metteur en scène) 


\author{
LE MONDE DU «SI» : \\ IDEOLOGIES INCERTAINES DANS \\ COMME IL VOUS PLAIRA *
}

Une relecture des Essais de Montaigne à la lumière du récent ouvrage d'Antoine Compagnon, Nous, Michel de Montaigne 1 est à l'origine de mon intérêt pour le monde incertain de Comme il vous Plaira. Par son analyse, Compagnon relie le scepticisme de Montaigne au nominalisme. Dans la comédie shakespearienne, le doute, l'inconsistance du nom, la vanité des mots font écho à bien des affirmations rencontrées dans les Essais, tels que précisément les essais intitulés "Des Noms», ou bien «De l'expérience ... ou "L'Apologie de Raymond Sebond": par exemple dans «l'Apologie» (II, xii) : «Toutes choses produites par nostre propre discours et suffisance, autant vrayes que fauces, sont subjectes à incertitude et débat ...»

"l'essence mesme de la vérité, qui est uniforme et constante, quand la fortune nous en donne la possession, nous la corrompons et abastardisons par nostre faiblesse»; ce qui conduit à la confusion, la confusion de la Tour de Babel, "qui vient essentiellement de l'emploi impropre ou abusif du langage». Et dans «De la gloire» (II, xvi, début): «il y a le nom et la chose; le nom ce n'est pas une partie de la chose ny de la substance, c'est une pièce étrangère joincte à la chose, et hors d'elle».

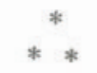

La confusion règne dans le monde qu'habitent Orlando et Rosalinde, le Duc Frédéric et Olivier, Jacques et le fou Touchstone, Pierre de Touche. Nul Pierre de Touche ne pourra jamais essayer le vrai et le faux dans l'enchevêtrement des hasards de la Fortune, des "complots déroutés» (Hamlet. V.ii. 395) et des attentes qui constituent cette illusion théâtrale de l'expérience de la vie. Assertions et dénégations s'affrontent dans l'atmosphère ambiguë d'une dialectique incessante. Il y a autant d'insécurité dans cette 
comédie que dans Le Roi Lear; rien n'y est sûr, ni pouvoir ni engagement; il est impossible de se fier aux mots; les voix des personnages aux résonances contradictoires se répondent à de muitiples nivaux.

Comme dans Le Roi Lear, les répétitions fréquentes du tout petit mot «si», «if», sont symptomatiques. Il reflète les hésitations, les humeurs changeantes. A Pierre de Touche qui proclame (V.iv. 100) «Il y a beaucoup de vertu dans un si», et explique que le «si» pourrait bien être le seul pacificateur, car il calme les querelles et porte en lui les arguments de la réconciliation, il est facile de rétorquer que le «si» peut être encore signe de division.

La fable de la comédie qui, dans son titre même Comme il vous plaira -- est une énigme, met en valeur l'emploi métaphorique du langage. Nous découvrirons surtout deux usages du "si» dans la comédie :

1. le «si» qui peut menacer par l'alternative, mais pourra aussi exprimer la condition susceptible de rendre la société cohérente, pourra refaire l'unité entre le nom et l'objet (dans la logique de l'ordre, en relation, nous le verrons, avec le nom du père d'Orlando, ou du père de Rosalinde).

2. le «si» du paradoxe - dans la logique nominaliste, où les contradictions deviennent possibles : dans le jeu de l'amour relatif au déguissement Rosalinde-Ganymède. Le «si» exprime alors la possibilité d'être autre : c'est l'impossible défi pour une femme d'être un homme ou pour un homme d'être une femme.

L'interrogation insistante d'Audrey (III. iii. 15-16) qui veut savoir ce que veut dire "poétique»: «Est-ce quelque chose d'honnête en action et en paroles ? Est-ce quelque chose de vrai ?» s'applique à beaucoup plus qu'à la poésie. La quête de la vérité, l'écart séparant les mots et les actes qui est l'écart qu'implique la métaphore, sont constamment suggérés. Shakespeare souligne l'échec du langage comme aussi ce que Montaigne appelle «La perpétuelle altercation et discordance d'opinions», cependant que tout au long des épreuves énigmatiques de la forêt, il conduit ses personnages qui ne cessent de poser des questions à se mettre eux-mêmes en question, les invitant à saisir leur identité. Cette quête de l'identité est l'un des intérèts majeurs de Comme il vous plaira. 


$$
\text { * } *
$$

«La question est de paroles», cette expression de Montaigne dans «De l'Expérience» résume l'impression d'ensemble que laisse la comédie dont l'univers est bruissant de rumeurs, sources de confusion. C'est comme La Maison de Rumeur d'Ovide ${ }^{2}$ où les mots passent d'un endroit à l'autre au gre du hasard, où l'apparence joue avec la réalité oủ les histoires vraies ou fausses s'amplifient ou se transforment. Les nouvelles s'y répandent selon l'habileté de celui qui parle ou les caprices de la Fortune :

Celia : Bonjour Monsieur le Beau, quelles nouvelles?

Le Beau : Belle princesse ! quel divertissement vous avez manqué !

Celia : Divertissement? de quelle couleur?

Le Beau : De quelle couleur, Madame ? Comment vous répondre ?

Rosalinde : Comme le voudront esprit et fortune.

Langage et fortune sont les mots-clés révélant l'instabilité d'une société dont les actes et les pensées sont fondés sur des oui-dire. A la question d'Olivier (I.i.99 et $s q q)$ : "peux-tu me dire si Rosalinde, la fille du duc, est bannie avec son père ?", Charles donne cette réponse convaincue : "Oh non, car sa cousine, la fille du nouveau duc ...l'aurait ... suivie dans son exil ou de douleur serait morte ... Le duc garde sa nièce à la cour et il ne l'aime pas moins que sa propre fille ...» Or cette affirmation est bientôt contredite (I. iii.) par le duc Frédéric qui bannit sa nièce : «si dans les dix jours tu n'es pas à vingt milles d'ici, tu mourras». Le "si» de l'alternative entre la vie et la mort, le "si» de la menace succède à la description d'un monde où le langage se dissociait du réel.

Les nouvelles changeantes montrent les réactions imprévisibles des personnages. A l'instabilité du duc Frédéric correspond la versatilité d'Olivier, qui déclare au sujet de son frère Orlando : «... j'espère en être bientôt débarrassé ... je ne sais pourquoi, mon âme le hait plus que tout. Pourtant, il est bien né ...» «je ne sais pourquoi», «pourtant»: nous remarquons l'ambiguité de cet autre mot «pourtant», "yet» ... très proche d'un «si».

Comme le dit Rosalinde, la Fortune aveugle compro- 
met les dons de la nature (I. ii. 30. et sqq). Et cette insistance sur le rôle de la Fortune est un point commun avec Montaigne : le nom d'un homme, son renom - c'est-à-dire, explique $\mathrm{A}$. Compagnon ${ }^{3}$, son nom répété - la réputation qu'on lui fait, sont soumis à la fortune. Il est dit que «La rumeur publique dore et redore les progrès» (1. i. 6) de Jacques, le frère d'Orlando, et Olivier aussi constate que «la rumeur publique» parle d'Orlando avec affection. Mais on observe vite selon ce que dit Montaigne (608 b) qu' «En cette confusion venteuse de bruits, de rapports et opinions vulgaires qui nous poussent, il ne se peut établir aucune route qui vaille».

Sur ce point Shakespeare partage l'intérêt de Montaigne pour le nominalisme. La philosophie d'Ockham et son scepticisme sur lesquels Montaigne fondait une part de sa méditation formaient, à la veille d'Hamlet ${ }^{4}$, tout un courant qui s'ajoutait au doute naturel et à l'autocritique des humanistes. La nature équivoque des noms, la vanité du renom illustrent le nominalisme de Montaigne. L'idée qu'un nom en soi n'existe pas in re mais seulement in voce. puisqu'il ne s'appuie que sur du vent, c'est-à-dire sur rien, est au coeur de son argumentation dans «De la Phisionomie» (III. xii) où il est écrit (1014b) que «les hommes ne s'enflent que de vent, et se manient à bonds, comme les balons».

La défiance à l'égard des noms, qui est évidente dans Roméo et Juliette (II. ii. 42) : quand Juliette dit à Roméo "C'est seulement ton nom qui est mon ennemi» ou (II.ii.42) avec la question : «Qu'y a-t-il en un nom ?» apparait dans divers incidents de Comme il vous Plaira, qui sont l'analyse et la critique du monde aveuglé dont le jugement repose sur de simples noms. Le duc Frédéric demande à Orlando (I. ii. 209) : «Quel est ton nom, jeune homme ?» Orlando répond :«Orlando ... le plus jeune fils de Sire Roland des Bois». Alors le duc : «J'aurais mieux aimé voir en toi le fils d'un autre/ Ta victoire m'aurait satisfait davantage/ si tu étais issu de maison différente». Lorsque dans la scène suivante, il bannit Rosalinde, parce que «tu es la fille de ton père ...» (I. iii), il donne à Celia une explication signifiante :

elle te vole ton renom.

Tu auras plus d'éclat et sembleras meilleure 
Si elle part d'ici ...

Tout comme l'argent qui devient papier ou signature sur un bon de garantie, le nom est fictif. Mais pour Frédéric, le fictif a une réalité solide.

La valeur fictive du nom est plus évidente encore, lorsque, quittant la cour pour la forêt d'Arden, Rosalinde décide de s'appeler Ganymède, s'attribuant le nom du page de Jupiter. Ce nom ne peut pas lui assurer le comportement d'un homme. Ganymède ne pourra souvent que "contrefaire» Rosalinde (IV. iii. 166), d'où la remarque d'Olivier, quand Rosalinde est prête à s'évanouir "Vous, un homme ! vous n'avez pas le coeur d'un homme», comme si un nom ne pouvait même pas être déguisement.

En tenant compte du point de vue nominaliste selon lequel les abstractions, les idées générales, les universaux n'existent pas en dehors des particuliers, et seul existe l'individuel, on comprend la distinction entre le nom et la personne que nous venons de souligner, distinction souvent à l'origine d'excellents effets dramatiques. Il y a dans Jules César (III. iii. 5 et sqq.) le cas extrême de Cinna le poète, massacré par la foule à cause de son nom.

Cinna : Je suis Cinna le poète.

Je ne suis pas Cinna le conspirateur. 4e plébéien :... Peu importe, ton nom est Cinna !

(et il est mis en pièces par la populace)

Et L'Alchimiste de Ben Jonson renferme l'épisode burlesque concernant l'anabaptiste (V. 71) Ananie, brutalement chassé parce qu'il porte le nom du coquin qui trompa les Apôtres (allusion à l'épisode raconté au chapitre ler du Ve livre des Actes des Apôtres.).

Que le même nom s'applique à différentes personnes prouve qu'il n'y a pas de différence entre noms propres et noms communs. Pour Montaigne, c'est la principale objection que l'on peut faire à ceux qui accordent trop d'importance à ce simple signe qu'est le renom. Le début de l'essai «Des Noms» (I, xi. vi.) explique non sans ironie :«quelque diversité d'herbes qu'il y ait, tout s'enveloppe sous le nom de salade ... " Pierre de Touche pourrait donc avoir raison, qui appelle Jacques : "Monsieur Qui que vous soyez ..." (III. iii. 67), comme si tous les noms étaient interchangeables. On pourrait même se demander si cela n'éclairerait pas le choix que fit Shakespeare du même nom pour deux 
personnages distincts de la Comédie, Jacques le philosophe et Jacques, le second fils de Sire Roland (ce qui, pour les critiques, est matière à plus d'une conjecture); mais sans doute serait-ce aller trop loin.

Il est aisé de passer de l'idée que les noms ne sont que voces à celle de l'arbitraire du langage, en relief dans les Essais, où apparait que «la pire ignorance est celle qui croit au langage». Le langage peut n'être qu'artifice, composé de mots gravés à l'intérieur des bagues (III. ii. 265-267). Souvent il abdique. Il arrive que «la chose ne se puisse dire en 'bloc' comme la pierre, en un mot» pour reprendre l'expression d'A. Compagnon ${ }^{5}$ qui s'inspire de Montaigne : la pierre (qui est aussi la maladie de Montaigne) étant le paradigme de l'objet dont l'existence est indubitable. La scène ii de l'acte III où Celia annonce à Rosalinde qu'elle a rencontré Orlando dans la forêt en offre l'amusante démonstration : les multiples questions de Rosalinde : ... que vaisje faire à présent de mon haut-de chausse ? Que faisait-il quand il t'a vue ? Que disait-il ? Quel air avait-il ? Où allaitil ? que fait-il ici ? T'a-t-il parlé de moi ? Où habite-t-il ? Comment t'a-t-il quittée ? Et quand dois-tu le revoir ? Réponds-moi d'un seul mot» provoquent cette réponse moqueuse de Celia : "Il te faudra alors me prêter la houche de Gargantua; car c'est un mot trop volumineux pour une bouche de notre temps».

Ailleurs, les mots ne peuvent pas dire l'inexprimable : le silence seul ou le geste peuvent exprimer la vérité. Orlando en donne l'exemple après que Rosalinde lui eut offert en souvenir la chaine qu'elle portait au cou :...

Quel trouble pèse ainsi lourdement sur ma langue?

Je n'ai pu lui parler, bien qu'elle le voulût ...

(I. ii. 236)

Cela fait penser à la réponse, que dans un autre contexte. Cordelia fait à la question de Lear : (I. i.)

Lear: Que saurez vous dire pour gagner un tiers plus opulent que celui de vos soeurs? Parlez.

Cor : Rien, Monseigneur.

Quant à Rosalinde, c'est l'éloquence de son silence même que lui reproche le duc Frédéric. (I. iii. 76) :

Et jusqu'à son silence et son air résigné touchent le coeur du peuple enclin à la pitié. 


$$
*
$$

Puisque le mérite compte plus que le nom, le juste emploi du langage au temps de la Renaissance signifie harmonie entre pensée et parole, entre parole et action. Pour la plupart, les tensions, les points culminants que nous discernons dans le déroulement de l'action de Comme il vous Plaira se rapportent au respect ou au non-respect de la parole solennelle, des serments, contrats, promesses vraies ou fausses.

Tout d'abord, si un nom n'a pas de substance, le renom - le nom répété -, la gloire, ne sont que signes équivoques. Dans la comédie, la Chanson des Seigneurs (IV. ii. 10-19) glorifiant les cornes du cerf, qui sont un cimier de famille, que "ton grand-père, puis ton père ont porté», tient lieu d'une plaisanterie édifiante sur le faux renom. Toutefois si le langage et le nom sont arbitraires, il y a dans les Essais comme dans la comédie, une limite à ce nominalisme : la conviction que le nom du père n'existe pas seulement in voce, mais in re, puisqu'il est évident dans la ressemblance des enfants avec leurs pères. C'est la pierre d'achoppement pour Montaigne, qui le contraint à revenir au réalisme. Dans «De la Ressemblance des enfans aux pères» (II. xxxvi-xxxvii), il montre qu'il est vain de renier son père et d'effacer son nom car ils demeurent dans ce que nous sommes. Ce respect de l'effigie du père, c'est le respect de l'image de Dieu en l'homme. Rappelons l'allusion d'Orlando à Olivier : souvenez-vous de "ce que Dieu fit, votre pauvre et indigne frère ...» (I. i. 31). Orlando dit aussi à Olivier : "J'ai en moi autant de mon père que vousmême» ... et il parle de "l'esprit de mon père ... qui est puissant en moi (I. i.)».

L'image du père est la seule essence véritable du nom. Dans la forêt, après qu'Orlando a révélé son nom à l'ancien duc banni, celui-ci conclut (II. 7) :

$\mathrm{Si}$ vous êtes bien le fills du bon Sire Roland,

Et mes yeux en font foi qui retrouvent en vous

Son portrait ressemblant,

Soyez dans la forêt le bienvenu .

L'orgueil du nom du père apparait aussi chez Ganymède-Rosalinde qui, dans la forêt, a rencontré le duc 
banni,son père : «Il m'a demandé de quelle famille j'étais : Je lui répondis qu'elle était aussi bonne que la sienne» (II.iv). La ressemblance - tel père tel fils - existe dans l'esprit et dans le comportement. Quand à la fin du Ve acte, Jacques de Bois apporte d'heureuses nouvelles aux exilés, il ajoute :

J'ai dit vrai

Et je m'en porte garant ...

Son serment est accepté parce que garanti par sa déclaration précédente :

Je suis le second fils du vieux Sire Roland (V.iv) $\mathrm{Ce}$ serment confirme les liens d'une société en train de retrouver son harmonie perdue; il correspond au "si» de «Si vous êtes bien le fils du bon Sire Roland», le «si» qui exprime la condition nécessaire pour rendre la société cohérente. L'harmonie retrouvée, c'est le dénouement idéal d'une action qui avait commencé par le mépris de la parole solennelle du père, de ses dernières volontés, bafouées par le fils aîné. Le non-accomplissement du testement - qui impliquait un engagement - devait provoquer les perturbations sociales qui suivirent : le mépris du frère par le frère qu'il réduisait à une condition inférieure à celle des animaux ("ses chevaux sont mieux soignés») constituait une violation de la bienséance, du «décorum» dont dépendaient l'ordre social et la justice. L'ordre ancien avait cédé la place à l'individualisme égoiste et à l'insécurité. Quand Olivier lance son insulte aux vieux serviteur Adam : «Va-t-en vieux chien», celui-ci rétorque ... «Dieu soit avec mon ancien maitre; ce n'est pas lui qui aurait parlé de la sorte» (I.i. 76-79).

Vers le tournant du XVIe siècle, "être honnête en paroles et en actes», pour reprendre l'expression d'Audrey (III. iii) représentait l'idéal traditionnel, alors qu'une nouvelle société venait confronter l'ancienne. Cet idéal était maintenant de plus en plus remplacé par les vices à la mode: flatterie, calomnie, vaine forfanterie, parjure ... La fonction du mot étant essentiellement sociale, la valeur morale et la valeur rhétorique du langage se confondaient, et à travers le jeu de la comédie on devine le souci d'un emploi correct du langage, qui évoque la réflexion de Thomas Nashe : $\mathrm{Ce}$ que nous pensons, exprimons-le, ${ }^{6}$ et ce que nous exprimons, pensons-le : que notre discours s'accorde avec notre vie", 
réflexion à laquelle Edgar fera écho à la fin du $R o i$ Lear (V. iii) : "Il nous faut dire ce que nous ressentons, non ce que nous devrions» (325).

Préter serment, tenir une promesse sont objets de préoccupations pour Celia et pour Rosalinde. Celia déclare (I. ii) : «Tout ce qu'il [le duc Frédéric, son père] a pris de force à ton père, je te le rendrai par affection pour toi, je le jure sur mon honneur. Et si jamais je manque à ma parole, je veux être changée en monstre». Après la victoire d'Orlando sur Charles, Celia s'adessant au vainqueur prophétise :

Si vous savez tenir vos promesses d'amour

Comme vous avez fait pour vos autres promesses, Votre maitresse aura du bonheur.

Telle est la charnière de la comédie, construite sur un «si». «Si vous savez tenir vos promesses d'amour ...» laisse espérer l'issue heureuse de la comédie d'amour. Mais ce "si» crée le suspense. L'importance de cette condition indispensable à l'harmonie est rehaussée par les moqueries de Pierre de Touche dans la même scène. Comme Celia,Pierre de Touche jure "sur mon honneur» et explique qui lui a appris ce serment (I. ii. 58-65) : «un certain chevalier qui jurait sur son honneur que les crêpes étaient bonnes et sur son honneur que la moutarde ne valait rien. Or moi, je maintiendrai que les crépes ne valaient rien et que la moutarde était bonne, et pourtant le chevalier ne faisait pas un faux serment». Avec plus d'un «si», Pierre de Touche démontre que si vous jurez par ce qui n'est pas, vous n'êtes pas parjure - serments et promesses étant question d'opinion, puisqu'ils dépendent de tant d'alternatives. Notons que la voix rusée du bouffon, qui peut compromettre l'honneur du duc Frédéric est réduite au silence. "Quelle pitié que les fous ne puissent parler des folies que font les sages». Tout est problème de point de vue. C'est «l'infinie et perpétuelle altercation et discordance d'opinions» dont parle Montaigne

La structure de la comédie repose sur une dialectique d'alternatives, exprimant des contradictions rendues acceptables, mettant ainsi en cause la logique de ce qui est un, la logique d'un monde unifié, et justifiant la multiplicité des opinions changeantes. Par exemple la vie dans la forêt revêt un aspect différent selon chacun de ses visiteurs : le duc banni y trouve douces les façons de l'adversité, il y écoute les leçons de l'arbre et du ruisseau qu'il préfère à la pompe 
de la cour; pour Jacques le mélancolique, cette vie en forêt n'est qu'usurpation tyrannique provoquant la mort des animaux innocents sur leur propre terrain. Quant à Pierre de Touche, plus il pénètre dans la forêt, plusil s'aperçoit qu'il est fou, mais il se résigne au contentement.

Montaigne dans «De l'expérience» (III. xiii) propose ce commentaire : "Jamais deux hommes ne jugeront pareillement la même chose, et est impossible de voir deux opinions semblables exactement, non seulement en divers hommes, mais en même homme». Le badinage de Rosalinde au sujet du temps qui «n'a pas la même allure pour tout le monde» reprend une idée semblable en une série d'images (III. ii) : «Le temps trotte avec la jeune fille entre son contrat de mariage et le jour de ses noces» (elle prend sept jours pour sept années), "Le Temps va l'amble avec un prêtre qui ne sait pas le latin et un riche qui n'a pas la goutte ...» L'expérience révèle la relativité de tout. Peut-être le meilleur passage sur l'idée du relatif est-il la conversation de Pierre de Touche et de Corin (III. ii. 11 et sqq) :

Corin: Et que vous semble cette vie de berger, maitre Pierre de Touche?

le Fou: A vrai dire Berger, si on la considère en soi, c'est une bonne vie, mais si on considère que ce n'est qu'une vie de berger, elle ne vaut rien ...

$\mathrm{La}$ veine satirique suggère le scepticisme de Pierre de Touche. L'argumentation de Corin en est l'illustration :

Cor: Vous me dites qu'à la cour on se baise les mains en guise de salut; cette courtoisie serait malpropre si les courtisans étaient bergers.

Pierre de Touche : la preuve !

Cor: $\quad$...nous sommes toute la journée à toucher nos brebis, et leurs toisons, vous savez, sont graisseuses.

Pierre de Touche : Mais les mains de nos courtisans, est-ce qu'elles ne suent pas ...? et la graisse d'un mouton n'est-elle pas aussi saine que la sueur d'un homme?

Justement Montaigne aime à dire : «...il y a plus de différence entre un homme et un autre qu'il n'y a de diversité entre un homme et un animal». Poussant l'argument jusqu'à l'absurde, il montre la vanité qu'il y aurait à classer les hommes selon leurs noms à l'exemple de l'empereur romain 
qui faisait "distribuer ... ses mets par la considération des premières lettres du nom des viandes : on servait celles qui se commençaient par M : comme mouton». ${ }^{7}$ Ainsi Pierre de Touche assimile la sueur du courtisan à la graisse du mouton. Shakespeare emploie précisément ici le mot «mouton» qui avait le sens de «sheep». Ce rapprochement amusant souligne l'indifférenciation de l'homme et de l'animal. Le nom de l'homme ne met donc pas en valeur son identité, et nous revenons à notre précédent propos sur le vide, le creux des noms auxquels on ne saurait se fier.

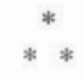

Dans le monde dramatique du «si» plusieurs effets comiques proviennent des confrontations de personnages et de mentalités contradictoires. Les différents couples d'amoureux illustrant différentes formes d'amour produisent des jeux combinés de perspectives qui font découvrir au spectateur ce "sujet merveilleusement vain, divers et ondoyant qu'est l'homme». L'expérience de chaque homme reste inachevée; la vérité peut toujours avoir un autre visage. Rien n'est définitif, tout est bigarré comme l'habit du fou. Pierre de Touche résume à peu près la condition de chacun dans son entretien avec William (Acte V. i. 20-32), ce William qui porte le même nom que Shakespeare, est né dans la forêt d'Arden et a aussi de l'esprit :

Pierre de Touche :

Tu t'appelles William?

William : William, Monsieur.

Pierre de Touche:

Un beau nom. Tu es né ici dans la forêt?

William : Oui, Monsieur, grâce à Dieu.

Pierre de Touche :

Grâce à Dieu; bien répondu. Es-tu riche ?

William: Ma foi, Monsieur, comme ci comme ça.

Pierre de Touche :

Comme ci comme ça est bien, très bien, tout ce qu'il y a de bien, et cependant ce n'est rien du tout, c'est seulement comme ci comme ça. Estu sage?

William : Oui, Monsieur, je suis suffisamment sage. 
Pierre de Touche :

Tu réponds bien. Je me souviens d'un dicton : «le fou croit qu'il est sage, mais le sage sait qu'il est fou ....

De méme ni la cour, ni la campagne, ni la vertu ni le vice ne peuvent être jugés bons ou mauvais.

Aucun jugement ne saurait être "constant et uniforme", qualités qui, nous dit Montaigne sont celles de la vérité; et Jacques n'aura pas de peine à chanter une chanson pour caricaturer celle d'Amiens, qui trouve le bonheur à l'ombrage des bois. Ce qui crée l'impression d'inachèvement des personnages, de leurs comportements toujours changeants, c'est qu'ils ont conscience de jouer des rôles qui varient comme au théâtre (II. vii. 7). Même Jacques ne se satisfait pas d'être figé dans l'attitude du mélancolique; il voudrait être bouffon, voire réformateur pour purger le corps corrompu de la société, alors que lui-même est l'image de la corruption de l'amour. Ne fut-il pas libertin et sensuel comme le dit le duc? (II. vii. 64-67). On se rappelle la mise en scène de Beno Besson où le personnage montrait sur son visage "les tumeurs, les horreurs purulentes", fruits de ses vagabongages. Le monde du "si» est celui de l'infinie variété de l'homme caméléon. Silvius et Phébé sont des acteurs jouant un rôle dans le spectacle qu'ils offrent à Rosalinde (III. iv), qui elle-même tient un rôle actif (III. iv. 5). Dans ce jeu de l'amour, aux clichés, aux attitudes affectées de Phébé, la cruelle bergère, s'oppose l'amour qu'éprouvait jadis Pierre de Touche pour Jane Smile (Jane Sourire) : «Je me souviens qu'un jour je caressai à sa place une cosse de pois qu'elle tenait à la main : j'en pris les deux moitiés et dis, les lui rendant, les yeux pleins de larmes, comme s'il s'était agi de précieux bijoux : "porte-les pour l'amour de moi» (II. iv. 42-51), ce qui implique bien un rôle consciemment joué.

C'est sur cet arrière-plan où se dessine tout ce qu'il y a de relatif dans les conventions du jeu de l'amour que sont imaginées les rencontres de Rosalinde et Orlando.

Leur jeu commence par les vers qu'Orlando grave sur l'écorce des arbres. Le mot écrit, comme dit Montaigne, est la mémoire du nom ${ }^{8}$. les mots qu'écrit Orlando parlent de l'errance des pèlerins, de serments violés (III. ii.); surtout ils se rapportent au nom de Rosalinde, ce nom que Jacques, dans son humeur revêche n'aime pas (III. ii. 256-258) : 
Jacques: C'est Rosalinde qu'elle s'appelle?

... Je n'aime pas son nom.

Mais le portrait qu'Orlando trace de sa dame met en question la vérité, l'essence même de ce nom :

La nature a condensé en elle

La joue d'Hélène, mais point son coeur.

La majesté de Cléopâtre

Ce qu'Atalante avait de mieux

La modestie de la triste Lucrèce.

Ainsi Rosalinde formée de toutes les qualités de

Fut conçue par la conjonction des astres

la nature

pour posséder de maints visages, yeux et coeurs

les traits les plus estimés (III. ii. 145-150)

On se rappelle la question de Roméo (III. iii. 106-107) :

Dans quel vil endroit de mon anatomie

mon nom loge t-il ?

Le jeu du "si» auquel jouent les deux jeunes gens part d'un point de vue nominaliste. Ce jeu à faire semblant, où Rosalinde passe pour Ganymède, mais joue à étre "pour Orlando" «sa vraie, vraie Rosalinde» (IV, i.) montre bien que le nom n'est qu'un masque. "Que diriez-vous maintenant, si j'étais votre vraie, vraie Rosalinde ?». C'est comme le renversement de la question plus habituelle : «Et si j'étais quelqu'un d'autre ?». Ce discours nécessite l'emploi d'un qualificatif qui est adjoint au nom et le «si» introduit ce qualificatif. Le double nom - Ganymède-Rosalinde accentue le rôle joué. Cependant, loin de fixer son personnage dans une attitude théâtrale, ce double nom donne libre cours à toutes les qualités qui sont en Rosalinde. L'idée de la mutation et de la confusion des noms lui fait évoquer en plaisantant la métempsycose de Pythagore :

je n'ai jamais été ainsi versifiée depuis

le temps de Pythagore, où j'étais un rat irlandais .. (III. ii. 171-172).

Comme l'observe Antoine Compagnon, la métempsycose qui est un thème développé dans "L'Apologie», est une variété du problème du nom, voire son motif fondamental : elle conserve l'âme - l'essence - à travers plusieurs corps et plusieurs noms ${ }^{9}$. Le jeu de Ganymède-Rosalinde est encore un jeu de langage, mais dans un tel contexte sur quel langage l'amour peut-il se fonder ? Peut-il être question de 
vérité en amour ? Au dire de Celia, la langue de Rosalinde "fait des écarts hors de propos». Rosalinde ne s'engage à suivre ni le point de vue de Gynamède, ni celui de Rosalinde. Elle sait être réaliste, ou satirique ou romanesque, mais elle ne voudrait pas plus qu'Orlando voir guérir son amour. C'est Celia, non Rosalinde, qui rit de l'inconstance en amour d'Orlando (III. iv). Quant à Rosalinde, ses questions révèlent sa passion angoissée :

Ros: Mais pourquoi a-t-il juré de venir ce matin et ne vient-il pas?

Celia (moqueuse) : Ah, certes, il n'y a chez lui aucune sincérité.

Ros : il n'est pas loyal en amour ?

Celia : en amour, oui, mais je ne le crois pas amoureux.

Ros : Tu l'as entendu jurer tant et plus qu'il l'était.

Celia : il était n'est pas il est ...

Et plus loin : «il fait des vers galants, dit des mots galants, fait de galants serments et les rompt galamment ...»

La remarque de Celia sur «était» qui n'est pas «est» ajoute l'allusion nominaliste habituelle au temps, qui contribue comme l'espace à l'instabilité de l'homme.

Comme Dante pour qui l'homme était «instabilissimum atque variabilissimum animal ${ }^{10}$ - le rapprochement est souligné par A. Compagnon --, Montaigne écrit aussi dans «De la Vanité» (III. ix. 941c) : «moy à cette heure et moy tantost, sommes bien deux». De même, Rosalinde met en garde Orlando contre les promesses imprudentes. Elle lui conseille de ne pas promettre pour «toujours, plus un jour». "Dites seulement pour un jour et supprimez toujours (IV. i. 135)». Impartiale, elle explique : "Les hommes sont Avril quand ils font la cour et Décembre une fois mariés; les filles sont Mai quand elles sont vierges, mais le ciel change dès qu'elles sont femmes", tant il est vrai que le même individu, la même personne peut varier selon les heures" (III. xiii «De l'expérience»). Ainsi en découvrant son propre amour, Rosalinde apprend progressivement à Orlando à se connaitre. En tant qu'individu, il ne peut appartenir à la catégorie des amoureux, correspondant à la description toute faite : "une joue creuse, que vous n'avez pas, un oeil cerné de bleu et enfoncé, que vous n'avez pas; une barbe négligée ... que vous n'avez pas ...» (III. ii. 358). Pourtant il est «très soigné dans $\mathrm{sa}$ mise, comme quelqu'un qui marque 
bien plus d'amour pour lui-même que pour n'importe qui ..." (III. ii. 360-363). Tout ceci s'oriente vers la leçon du Temple de Delphes, "Connais-toi», que Narcisse n'apprendra jamais.

En une sêrie d'escarmouches, Rosalinde continue allègrement à mettre au jour les paroles fausses, les mensonges flatteurs, qui conduisent la ronde de la vie. Elle réussit grâce à ce que Pierre de Touche appelle "cet enfant doué, l'intelligence» (III. iii. 12) et grâce à de "jolis serments», ceux "que l'on peut faire sans danger» (IV. i. 175). Alors la traversée de la forêt peut être libération.

Quand Celia raconte comment la première fois elle vit Orlando dans la forêt, c'est avec cette simple image (III. ii. 233-234) : «Il était là gisant de tout son long comme un chevalier blessé». Quand, à l'acte V (sc. iii. 107-108), Olivier se décrit tel qu'il était au moment où Orlando le sauva du serpent et de la lionne qui le menaçaient, il le fait en ces termes :

Un malheureux dans ses haillons, enveloppé

De ses très longs cheveux, dormait le dos à terre.

Ces deux images de deux hommes étendus au sol comme pour mourir sont les emblèmes de la mort symbolique que chacun d'eux doit endurer avant de pouvoir se connaitre. Alors Olivier proclame sa conversion : «C'était moi; mais ce n'est plus moi ...) (IV. iii. 137). Quant à Orlando il doit aussi passer par l'épreuve dans le jeu de l'amour pour pouvoir se remettre en question et trouver son identité. C'est seulement alors que Rosalinde arrêtera le jeu et que tous deux seront prêts à découvrir la réalité, à entrer dans le réel. Orlando et Olivier représentent les variations imprévisibles de l'homme "vain, divers et ondoyant» qui ne saurait s'engager avec assurance sur la voie incertaine de l'avenir.

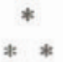

\section{Conclusion :}

Rosalinde est le magicien qui résout l'énigme et lui donne un dénouement rituel avec l'apparition d'Hyménéé. Comme il vous Plaira, qui a commencé dans la confusion avec le rejet des paroles solennelles d'un père, s'achève par 
la promesse heureuse de quatre mariages, promesse du renouvellement de la vie :

Paix ! assez de confusion

C'est moi, Hymen, qui vais conclure

Cette plus qu'étrange histoire;

Tels sont les mots du «dieu qui peuple toutes les villes». Pourtant rien encore n'est définitif. Phébé assure Sylvius "qu'elle ne reprendra pas sa parole», mais déjà Jacques peut prédire que la barque de Pierre de Touche et d'Audrey «n'a que pour deux mois de vivres». Il n'y a pas de sécurité pour la comédie de l'existence qui dépend d'un «si». «Si la vérité renferme le vrai...», tel est l'advertissement d'Hyménée. Même cette pirouette verbale n'est pas claire. Ces mots veulent-ils dire : «Si la vérité est vraie»? «Si les couples restent fidèles à leurs promesses» ? "à ce qu'ils ont prétendu être vrai» ? En tout cas, dans la vanité et la folie de ce monde du "si», qui est notre monde, le sceptique Montaigne parait avoir le dernier mot. Dans cette «branloire perenne» («Du reprentir»), y a-t-il quoi que ce soit que nous puissions connaitre ou prévoir ? Qu'est-ce qui est vrai ? Qu'est-ce qui n'est pas vrai ? Que pourrais-je dire ? Voilà l'interrogation constante de l'essayiste, qu'il aime à signifier par l'emblème d'une paire de balance (II. xii).

Avec l'épilogue, Rosalinde aussi captive les derniers instants d'attention de l'auditoire avec un "si» :

«Si j'étais vraiment une femme, j'embrasserais ceux d'entre vous qui ont des barbes qui me plaisent ...» «Si j'étais une femme» (les rôles féminins étaient tenus par de jeunes garçons) - le jeune garçon donc qui, sur la scène élisabéthaine jouait le rôle de Rosalinde, nous rappelle que son nom - le nom de Rosalinde - n'est bien qu'un masque, une simple voix de théâtre. 


\section{NOTES}

- - Ou Comment scepticisme, réalisme ou nom inalisme semblent dans la comédie être introduits par un «si»

- La traduction utilisé est celle de la Pléiade

1. Ouvrage publié par les Editions du Seuil, 1980.

2. Les Métamorphoses, Livre Xii.

3. A. Compagnon, Nous Michel de Montaigne, 1980, p. 103.

4. R. Quinones, The Renaissance Discovery of Time, 1972, p. 185.

5. A. Compagnon, ibid., p. 38

6. - The Anatomie of Absurditie, in Elizabethan Critical Essays, G. Gregory Smith, ed., Oxford Univ. Press, 1904, I, p. 335.

- Fgalement cité par T. McAlindon, Shakespeare and Decorum, 1973, p.6.

7. A. Compagnon, ibid, p. 58.

8. Ihid, pp. 98 et suivantes.

9. Ibid., p. 145 .

10. Ihid, p. 152 . 